\title{
Mesotelioma papilifero bem diferenciado do peritônio: relato de caso e revisão da literatura
}

\author{
Well-differentiated papillary mesothelioma of the peritoneum: case report and literature review \\ Daniela Batista de Almeida'; Daniel Meira Freitas'; José Alberto Nogueira²; Iguaracyra Barreto de Oliveira Araújoº
}

\begin{tabular}{|c|c|}
\hline unitermos & resumo \\
\hline $\begin{array}{l}\text { Mesotelioma } \\
\text { Patologia } \\
\text { Peritônio } \\
\text { Mesotelioma papilifero } \\
\text { bem diferenciado } \\
\text { Imuno-histoquímica }\end{array}$ & $\begin{array}{l}\text { Mesotelioma papilífero bem diferenciado (MPBD) é um tumor incomum, geralmente assintomático, } \\
\text { que pode ser encontrado incidentalmente no peritônio, apresentando-se como lesão nodular pequena } \\
\text { múltipla ou solitária. Apesar de ser considerado indolente, apenas uma minoria dos casos apresenta } \\
\text { sobrevida comprovada superior a dez anos. Relatamos um caso de uma mulher que, aos } 44 \text { anos, sem } \\
\text { história de exposição a asbesto, apresentou sintomatologia de ascite e obstipação com tumoração em } \\
\text { fundo de saco medindo } 8,3 \times 6 \times 5,6 \mathrm{~cm} \text {. A biópsia do tumor revelou morfologia compatível com MPBD. } \\
\text { Em função da impossibilidade de ressecção do tumor, a paciente foi submetida à quimioterapia. Evoluiu } \\
\text { com sobrevida de } 13 \text { anos. O diagnóstico diferencial com tumor seroso do ovário foi estabelecido através } \\
\text { da imuno-histoquímica (CK7+, Calretinina+, CK20-, CEA- e CD15-) e ultra-sonografia. Considerando } \\
\text { tamanho do tumor, impossibilidade de ressecção e sintomatologia, esse caso tem sobrevida superior } \\
\text { ao descrito na literatura. }\end{array}$ \\
\hline
\end{tabular}

abstract

Well-differentiated papillary mesothelioma (WDPM) is an uncommon tumor that can be found in peritoneum, usually found as an incidentally small nodular lesion, without symptoms. It is considered an indolent neoplasia, however only a minority of patients presents survival longer than ten years. This report concerns a case of a 44 years-old woman, without history of asbestos exposure; with complaints of ascites, constipation, and a mass in cul-de-sac measuring 5,6 $66,0 \times 8,3 \mathrm{~cm}$. The tumor biopsy revealed morphology compatible with WDPM. Due to the impossibility of resection, she was treated with chemotherapy and presents 13 years of survival. Differential diagnosis with serous tumor of ovary was established through imunohistochemistry (CK7+, Calretinin+, CK20-, CEA- and CD15-) and ultrassonography. Considering size of tumor, impossibility of resection and symptomatology, this case has superior survival than described in literature. key words Mesothelioma Pathology Peritoneum Well-differentiated papillary mesothelioma Immunohistochemistry

\section{Introdução}

Mesotelioma papilífero bem diferenciado (MPBD) é um tumor incomum que pode ser encontrado no peritônio, na pleura, no pericárdio ou na tunica vaginallis. Quando ocorre no peritônio é mais freqüente em mulheres de 30 a 50 anos de idade ${ }^{(5)}$. Diferentemente do mesotelioma maligno difuso, que tem uma ligação comprovada com a exposição ao asbesto, a associação etiológica do MPBD com este ainda não está estabelecida $^{(5,9)}$. Essa neoplasia usualmente manifesta-se como lesões nodulares pequenas, múltiplas ou solitárias, encontradas incidentalmente durante cirurgias ${ }^{(5,9)}$. Apenas uma minoria dos pacientes tem sintomas associados ao tumor $^{(9)}$. O MPBD geralmente apresenta comportamento indolente $\mathrm{e}^{(5,7-9,13)}$, porém a sobrevida além de

1. Estudante de Medicina da Universidade Federal da Bahia (UFBA)

2. Oncologista clínico do Serviço de Oncologia Clínica do Hospital Aristides Maltez.

3. Professora-adjunta, mestrado e doutorado, Departamento de Anatomia Patológica e Medicina Legal da Faculdade de Medicina da UFBA.

Trabalho realizado no Hospital Aristidez Maltez e apresentado no XXIV Congresso Brasileiro de Patologia (Florianópolis, abril de 2003) e no XXI Seminário Estudantil de Pesquisa em Salvador (dezembro, 2002). 
12 anos é rara. O acompanhamento por longo período é necessário em função do seu potencial em transformar-se em mesotelioma maligno difuso ${ }^{(9)}$.

Este relato refere-se a um caso de paciente do sexo feminino com 44 anos de idade, apresentando ascite e tumor irressecável em fundo de saco de Douglas. Ela foi submetida à quimioterapia e evoluiu com sobrevida superior a 13 anos.

\section{Relato do caso}

Em 1989, uma mulher de 44 anos foi admitida no nosso serviço em função de um aumento de volume abdominal e constipação. Ao toque vaginal identificou-se massa que ocupava o fundo de saco lateroposterior à direita. A ultrasonografia evidenciou volumosa tumoração sólida medindo 5,6 $6 \times 6 \times 8,3 \mathrm{~cm}$ ocupando fundo de saco posterior (Figura 1). A laparotomia exploradora revelou tumoração vegetante de $8 \mathrm{~cm}$ no fundo de saco de Douglas, irressecável, aderida ao reto e às alças intestinais, com útero e anexos livres. $\mathrm{O}$ exame anatomopatológico das secções da tumoração pélvica, coradas em $\mathrm{H} \& \mathrm{E}$, mostrou neoplasia constituída de proliferação de células cuboidais de núcleos arredondados, levemente hipercromáticos, formando projeções papilares em monocamada. Estas últimas exibiam eixos vascularizados, com presença de células inflamatórias e monócitos espumosos. Mitoses não foram visualizadas (Figura 2). Assim, obteve-se diagnóstico de MPBD.

A paciente fez uso de um ciclo de quimioterapia (CyVADACT - ciclofosfamida, vincristina, adriamicina e dactinomicina) e abandonou o tratamento. Permaneceu assintomática por quatro anos. Em 1996 voltou a apresentar ascite, constipação, além de edema e dor em membro inferior direito. Foi novamente submetida a tratamento quimioterápico, realizando seis ciclos de vincristina, durante o período de 1996 a 1997. Após o tratamento houve alívio da dor em membro inferior direito e a ascite passou a ter progressão lenta. A paciente passou a procurar serviço médico periodicamente para realizar paracenteses.

Em 2002 foi readmitida com ascite volumosa, e a ultrasonografia revelou duas tumorações pélvicas sólidas $(7,7 \mathrm{x}$ $5,7 \mathrm{~cm}$ e $8,3 \times 6,1 \mathrm{~cm}$ ), além de ascite de septos grosseiros (Figura 3). Em função desta progressão a paciente foi submetida à segunda laparotomia, que revelou tumoração vegetante recobrindo a pelve, esboçando aspecto de pseudocisto com papilas projetadas para o seu interior. Não foi possível identificar útero e anexos. Realizada nova biópsia, o material foi submetido a estudo histológico convencional e estudo imuno-histoquímico. Este último revelou células revestindo eixos conjuntivos fortemente positivas para citoqueratina-7 e calretinina (Figuras 4 e 5) e negativas para citoqueratina-20, CEA, BerEP4 e CD15 (Tabela 1). O índice de proliferação celular pelo MIB-1 foi menor que $1 \%$.

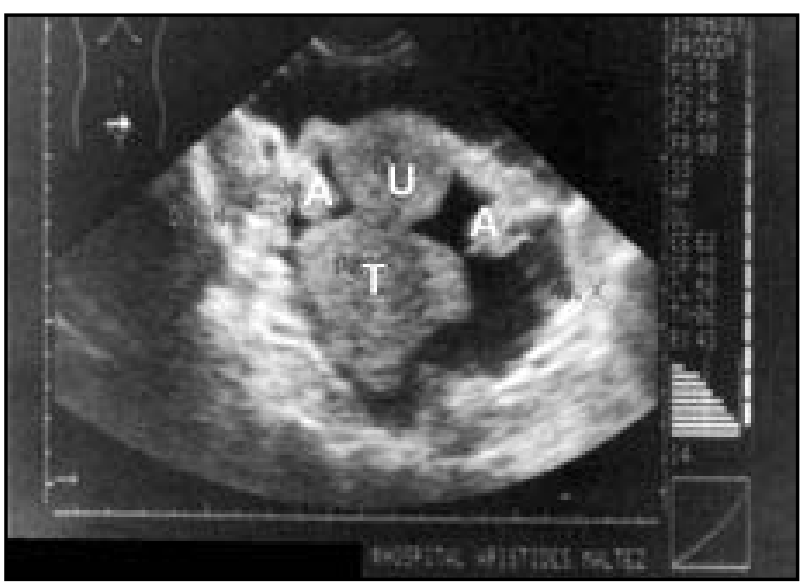

Figura 1 - Ultra-sonografia pélvica em 1989 mostrando massa tumoral (T) ocupando fundo de saco de Douglas, útero $(U)$ e anexos $(A)$ livres

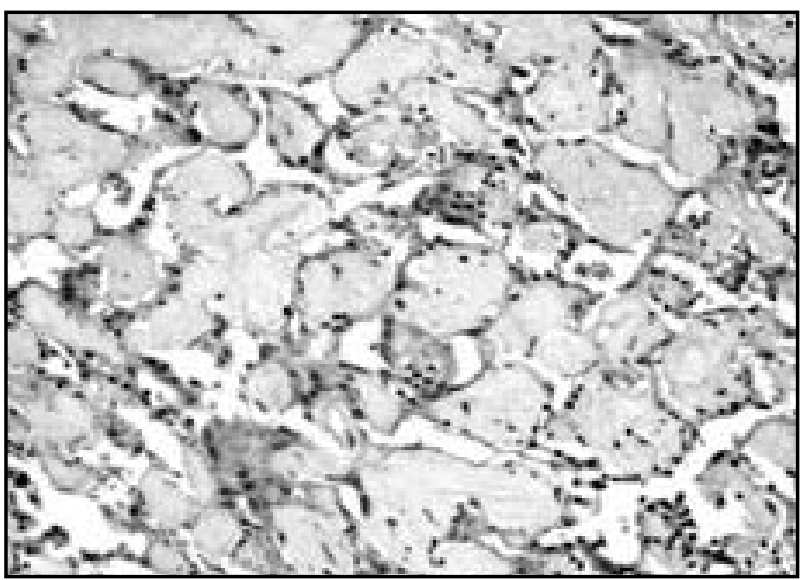

Figura 2 - Aspecto microscópico (H\&E) papilífero sem atipias celulares

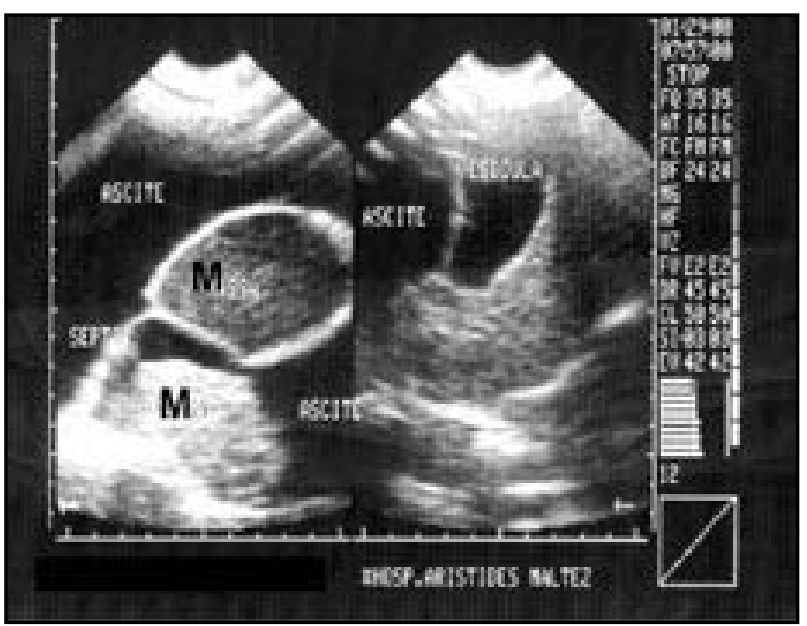

Figura 3 - Ultra-sonografia pélvica em 2002 mostrando duas massas (M) tumorais unidas por septo ocupando toda a pelve. Útero ou anexos não são identificados 


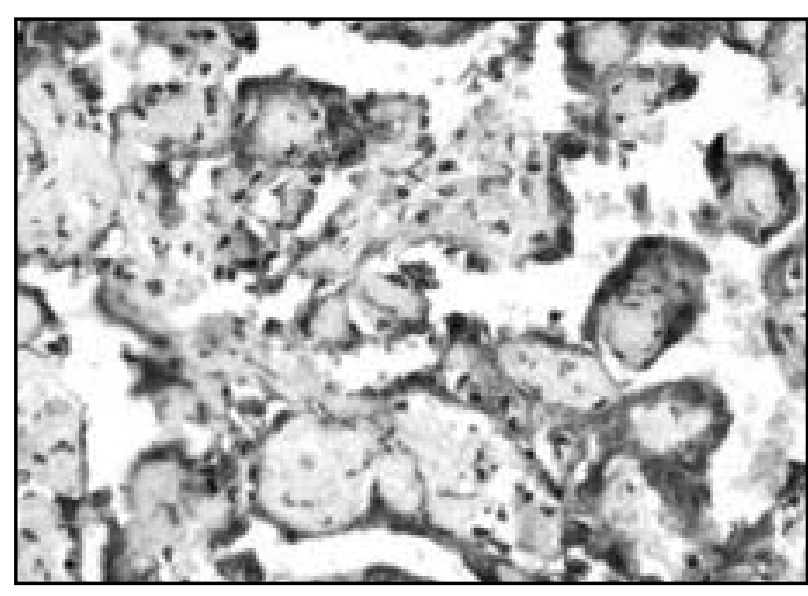

Figura 4 - Forte expressão de citoqueratina 7 em células mesoteliais

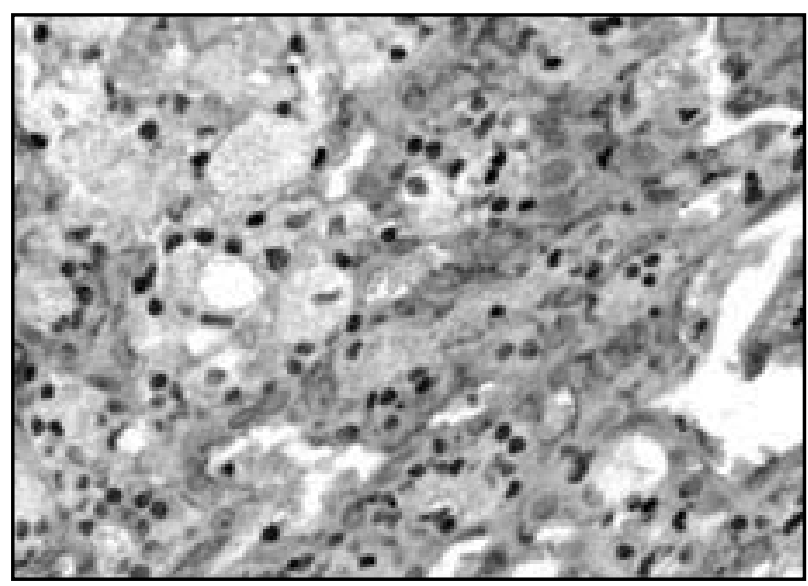

Figura 5 - Expressão de calretinina em cordões de células mesoteliais

\begin{tabular}{lccccccc} 
& & \multicolumn{6}{c}{ Resultados da imuno-histoquímica } \\
Tabela 1 & no MPBD & & & \\
\hline & CK7 & CK20 & CEA & CAL & CD15 & BerEP4 \\
Caso & + & - & - & + & - & - \\
\hline
\end{tabular}

MPBD: mesotelioma papilifero bem diferenciado; CK7: citoqueratina 7;

CK20: citoqueratina 20; CEA: antígeno carcinoembrionário; CAL: calretinina.

\section{Discussão}

Neste trabalho relatou-se o caso de uma mulher de 44 anos, apresentando tumor de 5,6 $66 \times 8,3 \mathrm{~cm}$ em fundo de saco de Douglas, irressecável, diagnosticado como MPBD. A paciente foi tratada com quimioterapia, evoluindo com ascite recorrente durante 13 anos. Atualmente encontra-se viva com doença.

Foram achados 41 casos de MPBD do peritônio na literatura ${ }^{(2-5,7-9)}$, acometendo predominantemente o sexo feminino (65,9\%). Do total, $24(58,5 \%)$ encontravam-se entre a terceira e a quinta década de vida, com a média de idade de 46,1 anos $( \pm 13,65)$, variando de 25 a 84 anos. Na maioria dos casos (55\%), o MPBD foi um tumor assintomático, descoberto incidentalmente. Quando presente, a sintomatologia relatada constituiu-se de dor abdominal $(38,8 \%)$, ascite $(33,3 \%)$, massa pélvica $(11,1 \%)$, doença pélvica inflamatória crônica $(11,1 \%)$ ou constipação $(5,5 \%)$. No nosso caso, tratava-se de um tumor sintomático que levou à intervenção cirúrgica.

O MPBD pode surgir em diferentes locais e, muitas vezes, em múltiplos sítios no mesmo indivíduo. As lesões dessa neoplasia geralmente consistem em nódulos pequenos, múltiplos ou solitários. À exceção do caso descrito por Bouvier et al.(3), os tumores variam de $0,5 \mathrm{a} 3 \mathrm{~cm}$ de diâmetro. Entre os locais citados na literatura, 26\% das lesões surgiram na parede de órgãos abdominais ou pélvicos; $22 \%$, no omento; $16 \%$, na parede pélvica; $14 \%$, no mesentério e; $14 \%$, no peritônio. A localização em fundo de saco de Douglas, como aqui relatada, ocorreu apenas em quatro (8\%) casos, sendo que apenas em um o tumor atingiu dimensões semelhantes.

O MPBD é considerado um tumor borderline, e é comum a sobrevida acima de cinco anos após o diagnóstico $(54,5 \%)$. Entretanto, raros casos apresentam sobrevida igual ou superior à referida neste trabalho (12\%). Após análise dos dados da literatura, não foi encontrada associação entre sobrevida e tamanho do tumor, tipo do tratamento ou sintomatologia do paciente. Apesar de ter comportamento indolente na maioria das vezes, descreveu-se na literatura a possibilidade de transformação em mesotelioma maligno difuso(4).

As complicações relatadas, decorrentes da neoplasia, foram torção do tumor, determinando quadro de abdome agudo, e compressão de estruturas adjacentes ${ }^{(5,7,8,13)}$. Relatou-se também um caso de disseminação da massa para 0 pericárdio ${ }^{(3)}$. Todas foram observadas em tumores extensos. Sendo assim, o caso aqui apresentado difere pela evolução sem intercorrências, apesar das elevadas dimensões.

Geralmente, tratamentos agressivos para MPBD não são indicados, sendo que a ressecção cirúrgica foi método de escolha em todos os 41 casos. Não houve tratamento adjuvante na maioria dos relatos. Analisando os dados disponíveis de 32 casos, procedeu-se com radioterapia em $18,8 \%$ e quimioterapia em $25 \%$ deles. Geralmente, o esquema de quimioterapia utilizado foi cisplatina e/ou doxorrubicina. Entretanto, dados de avaliação da eficiência desses métodos são precários. Segundo Daya et al. (7), o tra- 
tamento quimioterápico deveria ser reservado a pacientes com progressão evidente da tumoração. Em nosso caso, em função da impossibilidade de ressecção cirúrgica, foi utilizada somente a quimioterapia. Esta atenuou a sintomatologia, com desaparecimento da dor em membro inferior e da ascite, pelo menos por um período de quatro anos.

Diante da localização da neoplasia em fundo de saco e das características histopatológicas, células agrupadas em conformação papilar, foi necessário estabelecer o diagnóstico diferencial entre MPBD e tumor seroso da superfície do ovário (TSSO). A histologia evidenciando células cuboidais em monocamadas, sem mitoses ou anomalias celulares, sugere mesotelioma (Tabela 2). O estudo imuno-histoquímico revelou positividade para calretinina, um anticorpo que marca células mesoteliais em $60 \%$ a $100 \%$ dos casos e que raramente pode marcar adenocarcinomas $(0 \text { a } 28 \%)^{(10)}$. Além disso as células da neoplasia aqui relatada foram negativas para CD15 e BerEP4, anticorpos que marcam células epiteliais e que não estão presentes no mesotelio$\mathrm{ma}^{(10-12)}$. Entretanto, a sensibilidade do marcador CD15 é baixa, uma vez que ele é positivo somente em $30 \%$ a $80 \%$ dos TSSO11. O imunorreagente CEA, freqüentemente positivo em carcinomas pulmonares e gastrointestinais, é encontrado em apenas 0 a $35 \%$ dos carcinomas serosos do ovário(12). Portanto, a negatividade para esse marcador não auxilia na diferenciação entre mesotelioma e TSSO. A possibilidade deste último foi afastada em função de estudo imuno-histoquímico compatível com MPBD (Tabela 3), ultra-sonografia e laparotomia, evidenciando ovários livres inicialmente. Outro diagnóstico diferencial possível para este caso, em função da localização em fundo de saco, é tumor metastático, geralmente do trato gastrointestinal. Entretanto, a negatividade para CEA e CD20 desfavorece essa possibilidade e corrobora o diagnóstico de $\mathrm{MPBD}^{(12)}$.

Neoplasias envolvendo o peritônio são comuns na prática do patologista, principalmente as de origem metastática. O conhecimento acerca das neoplasias primárias do peritônio, relativo às possíveis formas de apresentações clínicas e caracterização histológica e imuno-histoquímica é fundamental para o diagnóstico diferencial com neoplasias metastáticas. O estudo imuno-histoquímico utilizando um painel amplo é necessário, uma vez que os marcadores têm positividade variável nas diferentes séries descritas na literatura. Neste trabalho relatou-se um caso de MPBD em fundo de saco com apresentação clínica distinta, cujo estudo imuno-histoquímico confirmou a origem mesotelial do tumor.

\section{Comparação das caracteristicas}

Tabela 2 histológicas de MPBD e TSSO

\begin{tabular}{lcc}
\hline & MPBD & TSSO \\
Células & Cuboidais & Cilíndricas \\
Disposição celular & Monocamada & Pluricamadas \\
Anomalias nucleares & Ausente & Presente \\
Mitoses & Ausente & Presente \\
\hline
\end{tabular}

TSSO: Tumor seroso da superfície do ovário.

Painel imuno-histoquímico comparativo: tumor seroso

Tabela 3 do ovário e mesotelioma

\begin{tabular}{lcc}
\hline Anticorpo & TSSO & Mesotelioma \\
CD15 & $+/-$ & - \\
Calretinina & $\mathrm{R}$ & + \\
BerEP4 & + & - \\
CEA & $-/+$ & - \\
CK7 & + & + \\
CK20 & - & - \\
\hline
\end{tabular}

+: quase sempre fortemente positivo, com reatividade difusa; +/-: predominantemente positivo; -/+: predominantemente negativo; -: quase sempre negativo; $R$ : raras células podem ser positivas.

\section{Referências}

I. ATTANOOS, R. et al. Value of mesothelial and epithelial antibodies in distinguishing diffuse peritoneal mesothelioma in females from serous papillary carcinoma of the ovary and peritoneum. Histopathology, v. 40, n. 3, p. 237-44, 2002.

2. BERGHOLZ, M.;ALTMANNS BERGER, M.; SCHAUER,A. Benign mesothelioma of the cul-de-sac. A tumor with misleading histologic pattern in an unusual localization. Gynecol Oncol, v. II, n. 3, p. 393-5, 1981.
3. BOUVIER, S. et al.Well-differentiated papillary mesothelioma of the peritoneum: an attenuated malignant tumor. Review of the literature a propos of a case. Bull Cancer, v. 81, n. 2, p. 104-7, 1994

4. BURRIG, K.; PFITZER, P.; HORT W. Well-differentiated papillary mesothelioma of the peritoneum: a borderline mesothelioma. Report of two cases and review of literature. Virchows Arch A, v. 4 I7, n. 5, p. 443-7, 1990. 
5. BURTNOR, K. J. et al.Well-differentiated papillary mesothelioma. Am J Surg Pathol, v. 25, n. I0, p. I304-9, 2001.

6. CHURG, A. et al. The separation of benign and malignant mesothelial proliferations. Am J Surg Pathol, v. 24, n. 9, p. I | 83-200, 2000.

7. DAYA, D.; MCCAUGHEY, W.T. E. Well-differentiated papillary mesothelioma of the peritoneum: a clinicopathologic study of 22 cases. Cancer, v. 65, n. 2, p. 292-6, 1990.

8. GOEPEL, R. J. Benign papillary mesothelioma of peritoneum: a histological, histochemical and ultrastructural study of six cases. Histopathology, v. 5, n. I, p. 2 1-30, 198 I.

9. HOEKMAN, K. et al. Well-differentiated papillary mesothelioma of the peritoneum: a separate entity. Eur J Cancer, v. 32, n. 2, p. 255-8, 1996.
10. ORDÓÑES, N. Immunohistochemical diagnosis of epithelioid mesotheliomas: a critical review of old markers, new markers. Hum Pathol, v. 33, n. 10, p. 953-67, 2002.

I I. ORDÓÑES, N. Role of imunohistochemistry in distinguishing epithelial peritoneal mesotheliomas from peritoneal and ovarian serous carcinomas. Am J Surg Pathol, v. 22, n. 10, p. 1203-14, 1998.

12. SOSLOW, R.; ISACSON, C. Diagnostic immunohistochemistry of the female genital tract. In: DABBS, D. Diagnostic immunohistochemistry. Philadelphia: Churchill Livingstone, 2002. p. 486-5I 6.

13. YOO, S. et al. The difficult diagnostic approach of the intraperitoneal mesothelioma. Neth J Med, v. 57, n. 6, p. 224-8, 2000. 